\title{
Chlorhexidine gel reduces incidence of alveolar osteitis after extraction of the mandibular third molars
}

\author{
Abstracted from \\ Hita-Iglesias P, Torres-Lagares D, Flores-Ruiz R, Magallanes-Abad N, \\ Basallote-Gonzalez M, Gutierrez-Perez JL. \\ J Oral Maxillofac Surg 2008; 66: 441-445

\section{Question: Is chlorhexidine gel more effective than chlorhexidine rinse in reducing alveolar osteitis in mandibular third molar surgery?}

Effectiveness of chlorhexidine gel versus chlorhexidine rinse in reducing alveolar osteitis in mandibular third molar surgery.

Address for correspondence: Dr Hita-Iglesias, University of Michigan, Oral and Maxillofacial Surgery, 1500 Medical Center Drive, Ann Arbor MI 48109-0018, USA. E-mail: pilarh@umich.edu; danielt|@us.es
Design A randomised, prospective clinical trial with parallel groups was carried out in a single centre.

Intervention The experimental (gel) group $(\mathrm{n}=41)$ applied a bioadhesive $0.2 \%$ chlorhexidine gel to the wound during the first postoperative week and a control (rinse) group ( $n=32)$ used a $0.12 \%(v / v)$ chlorhexidine mouthrinse during the first week following third molar extraction. Patients were evaluated on the third and seventh postoperative day. Outcome measure Alveolar osteitis was evaluated according to Blum's criteria..

Results A 70\% decrease in postoperative alveolar osteitis in the gel group (P 0.04) was observed. The rinse group had $25 \%$ incidence of postoperative alveolar osteitis, whereas the gel group had $7.5 \%$. T equates to a number needed to treat of six ( $95 \%$ confidence interval, 3-144).

Conclusions It was concluded that the topical application of bioadhesive chlorhexidine gel to the surgical wound during the postoperative week may decrease the incidence of alveolar osteitis after extraction of the mandibular third molars.

\section{Commentary}

Alveolar osteitis or dry socket is one of the most common postoperative complications following the routine extraction of permanent teeth, with a reported incidence of $3-4 \%$. The reported incidence of alveolar osteitis after the removal of impacted mandibular third molars is much higher, ranging from $1-45 \%$. This variability is probably caused by differences in diagnostic criteria and in the methods of assessment, as better controlled studies report an incidence of $25-30 \%{ }^{1}$

The aetiology of alveolar osteitis is still poorly understood, with many causative and precipitating factors implicated. These include bacterial infection, trauma during surgery, poor local blood supply, female hormones and the use of the oral contraceptive pill, and smoking. Current understanding is that the aetiology of alveolar osteitis is probably multifactorial but that aggravating or predisposing factors, particularly smoking and the use of the oral contraceptive pill, lead to an increased incidence.

This paper by Hita-Iglesias and colleagues is the second published by this group on the use and effectiveness of chlorhexidine gel in reducing the incidence of alveolar osteitis. The group's initial investigation was a randomised double-blind placebo-controlled study on the use of chlorhexidine gel after the removal of impacted mandibular third molars. ${ }^{2}$ Either chlorhexidine gel or a placebo gel was inserted into the extraction socket after the impacted mandibular third molar had been removed. The study was designed to ensure that there was an equal distribution of male and female patients, smokers and women taking the oral contraceptive pill in the study and control groups. The incidence of alveolar osteitis was evaluated using diagnostic criteria standardised by Blum. ${ }^{1}$ The study found that the incidence of alveolar osteitis in the control group was $30 \%$, as might be expected, but only $11 \%$ in the study group: a significant reduction (P 0.019). The study concluded that chlorhexidine gel applied once to the extraction socket significantly reduced the incidence of alveolar osteitis after mandibular third molar removal.

The aim of this study was to compare the effectiveness of chlorhexidine gel applied topically to the surgical wound with that of a chlorhexidine mouthrinse $(0.12 \% \mathrm{v} / \mathrm{v})$ in reducing postoperative alveolar osteitis after mandibular third molar removal. Several studies have reported the effectiveness of rinsing with chlorhexidine on the day of surgery and on the days following surgery in reducing the incidence of alveolar osteitis. ${ }^{3}$ Therefore it was a very reasonable question to ask.

The study reported a significant decrease of 30\% (P 0.040) in the incidence of postoperative alveolar osteitis in the topical chlorhexidine gel group, which had an incidence of only $7.5 \%$, compared with an incidence of alveolar osteitis of $25 \%$ in the chlorhexidine rinse group. The results reported in this study are not what would be expected, however. Chlorhexidine gel was inserted into the third molar extraction socket in both study groups. The sockets were sutured and the patients were then randomly allocated into one of the two study groups. In the first study group, the participants applied chlorhexidine gel topically to the surgical wound twice a day (morning and night) for a week and in the second group, individuals rinsed with chlorhexidine twice daily (morning and night) for a week. The authors conclude that the reduced incidence in the group that applied chlorhexidine gel was because of the prolonged release of chlorhexidine from the daily gel application.

The authors of the study fail to explain or discuss the high incidence $(25 \%)$ of alveolar osteitis in people who had chlorhexidine gel inserted into their extraction socket and then rinsed with $0.12 \%(\mathrm{v} / \mathrm{v})$ chlorhexidine twice daily for a week. 
The authors' previous study had reported an incidence of alveolar osteitis of only $11 \%$ when the chlorhexidine gel was placed in the extraction socket and, with the additional benefit of a chlorhexidine mouthrinse, the incidence alveolar osteitis would have been expected to be have been even lower, not $25 \%$ as reported.

One explanation could be the sample size, there being too few patients entered into the study. Forty-one patients comprised the topical chlorhexidine gel group, with only 32 people in the chlorhexidine rinse group. In view of the unexplained results of the study, the conclusions should be viewed with discretion.
Keith G Smith

Oral and Maxillofacial Medicine and Surgery, University of Sheffield, School Of Clinical Dentistry, Sheffield, UK

1. Blum IR. Contemporary view on dry socket (alveolar osteitis); a clinical appraisal of standardization, aetiopathogenesis and management: a critical review. Int J Oral Maxillofac Surg 2002; 31: 309-317.

2. Torres-Lagares D, Gutierrez-Perez J, Infante-Ccossio P, Garcia-Calderon M, RomeroRuiz MM, Serrera-Figallo M. A randomised, double-blind study on the effectiveness of intra-alveolar chlorhexidine gel in reducing the incidence of alveolar osteitis in mandibular third molar surgery. Int J Oral Maxillofac Surg 2006; 35: 348-351.

3. Caso A, Hung LK, Beirne OR. Prevention of alveolar osteitis with chlorhexidine: a metaanalytic review. Oral Surg Oral Med Oral Pathol Oral Radiol Endodontol 2005; 99: 155-159.

Evidence-Based Dentistry (2009) 10, 54-55 doi:10.1038/sj.ebd.6400655 